\title{
Mixité et inégalité du défrisage chez les femmes et hommes en France et au Cameroun
}

\section{Eliane Eock Laiifa*}

\section{Résumé}

Cet article propose une analyse critique de la pratique du défrisage chez les femmes et les hommes au Cameroun et en France. L'enquête de terrain menée entre 2011 et 2014 en France et au Cameroun auprès d'une centaine de femmes et d'hommes démontre d'une part la mixité de cette pratique, d'autre part des différences dans son traitement et son appréciation en fonction du sexe. Enfin, les résultats révèlent une inégalité en défaveur des femmes et confortent la théorie des contraires (Synnott, 1987) qui opposent notamment pilosités masculine et féminine. Le poids du stigmate «cheveu crépu» pèse plus lourdement sur elles que les hommes en raison même de leur sexe.

Mots-clés : défrisage, identité, théorie des contraires, pilosité, rituel.

\footnotetext{
*Université de Strasbourg, Strasbourg, France.
} 


\section{Gender diversity and disparity in hair straightening among women and men in France and Cameroon}

\section{Abstract}

This article provides a critical analysis of the practice of hair straightening among women and men in Cameroon and France. The field survey, carried out between 2011 and 2014 in both countries, which included a sample of one hundred women and men, demonstrates, on the one hand, a sex balance in this practice; on the other hand, it shows some differences based on sex in its processing and appraisal. Finally, the results reveal some inequality detrimental to women and confirm the theory of opposites that confront, for example, male and female body hair (Synnott, 1987). The weight of the "frizzy hair" stigma bears more heavily on women than on men just because of gender differences.

Keywords: hair straightening, identity, opposition, body hair, ritual.

\section{Introduction}

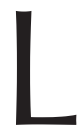

a modification du corps en général, celle des cheveux en particulier, s'inscrit dans différents domaines de la sociologie et de l'anthropologie sociale et culturelle. Je retiens ici deux qui apparaissent pertinentes dans le contexte de cet article ${ }^{1}$. D'une part, les techniques du corps, ou pratiques esthétiques pour être plus précise, telles qu'établies par Mauss ([1950] 2013). Dans cette perspective, le corps est notre premier outil (Mauss [1950] 2013). Il est à la fois «objet et instrument d'une culture» (Saliba, 1999, p. 4) qu'il concourt à construire symboliquement. Le corps produit et reproduit la culture. II la matérialise et I'actualise dans I'espace et dans le temps. Le corps réactualise la culture tout en la modifiant, il en confirme des parties tandis qu'il en abandonne d'autres. «Ces techniques sont donc les normes humaines du dressage humain» (Mauss [1950] 2013, p. 374). En ceci, elles

\footnotetext{
${ }^{1}$ En s'en tenant strictement au défrisage, nous avons une troisième catégorie, les black studies. Mais je reviendrai sur ce point dans la conclusion. M'intéressant aux deux sexes, j'exclue provisoirement le féminisme noir, black feminism afin de ne pas m'éloigner de mon propos
} 
participent par conséquent à la socialisation et à l'intégration de l'individu dans un(des) groupe(s) donné(s). Les modifications corporelles y compris capillaires situent l'individu dans un contexte esthétique, sociologique, culturel, historique, économique, politique, etc.

D'autre part, les modifications corporelles, ici capillaires, s'inscrivent dans l'étude du genre, gender studies. Le même phénomène, ici le défrisage, est conçu, perçu, analysé différemment en fonction du genre du pratiquant. Le sexe est primordial dans le processus de socialisation et d'intégration de I'individu à sa société en ce qu'il permet une différenciation entre deux classes sexuelles. Il s'agit là de la première des divisions, presqu'à caractère ontologique puisque que cette partition homme-femme, mâle-femelle traverse toutes les sociétés et qu'elle imprègne nos imaginaires collectifs comme individuels. Les traitements capillaires, bien que mixtes, participent de cette socialisation différentielle et de la domination masculine. Ils sont un produit évident et un instrument de la domination masculine à l'égard des femmes.

S'appuyant sur une ethnographie conduite en France et au Cameroun (2011-2014), cet article interroge le défrisage en tant que pratique capillaire illustrant notamment I'opposition homme/femme selon la théorie des contraires de Synnott (1987). En effet, l'examen de certains éléments de cette pratique régulière y révèle des différences en fonction du sexe qui illustrent l'inégalité sexuelle face au stigmate du cheveu crépu. Dans un premier temps, l'article présente une ethnographie du défrisage en tant que pratique mixte, mais différenciée. Pour cela, nous examinons quelques éléments en lien avec cette pratique, tels que l'âge du premier défrisage et leur fréquence. Dans un deuxième temps, nous envisageons les soucis exprimés et l'opinion qu'ont les femmes et hommes défrisés sur les cheveux crépus. Nous déterminerons ainsi dans quelle mesure cela concourt, entre autres avec les précédents éléments, à maintenir une inégalité de traitement selon le sexe.

Il est possible de détendre ou de lisser une chevelure crépue grâce à différentes techniques telles que le brushing. Le défrisage à froid reste le plus répandu selon les chiffres de l'industrie capillaire. II implique l'usage d'un type de crème relaxante qui modifie définitivement la structure des 
cheveux. Son usage participe de la stigmatisation des chevelures crépues (Sméralda, 2004 ; 2012).

Les Afro-américains, suivis par d'autres populations noires, rejettent le défrisage dans les années 1950-1960, lors de La Lutte pour les Droits civiques. Ils le critiquent et l'abandonnent un temps en faveur de la coupe afro $^{2}$. Elle symbolise alors leur désir d'africanité, d'une identité noire valorisée (Eyene, 2008). Mais après les années 1970, I'afro perd de son attrait et le défrisage se démocratise et se répand (Sméralda, 2004) même si depuis les années 2000, le mouvement nappy ${ }^{3}$ exhorte les femmes noires à y renoncer afin de se réapproprier leur chevelure crépue (Herron, 1998 ; Wilkerson, 2017) afin de l'imposer dans l'espace public où il est encore indésirable (Sméralda, 2012). Ce mouvement révèle la stigmatisation des cheveux crépus dont les femmes sont les principales victimes, mais aussi la pression sociale qui les incite au défrisage.

Selon mes recherches (voir aussi Synnott, 1987), les femmes s'identifieraient plus à leurs cheveux que les hommes, ce qui confère au stigmate du cheveu crépu un plus grand pouvoir. La beauté ferait et définirait la femme (Bernuzzi Sant'Anna, 1995 ; Le Breton, [1990] 2011). Cumulant féminité et cheveux crépus, les femmes noires aux boucles serrées subissent une double injonction, être féminine et avoir de longs cheveux lisses, en un mot, être belle selon des critères occidentaux.

La belle femme noire qui réussit à une peau nettement plus claire que celle des hommes et de longs cheveux lisses (Bromberger, 2010). Cependant, tandis que le défrisage féminin est à la fois valorisé et stigmatisé, le défrisage masculin est ignoré ou minoré, marginalisé. Cela concorde avec la théorie des contraires selon laquelle les sexes opposés ont des pilosités opposées, les cheveux et les poils du corps sont opposés, les idéologies opposées ont des pilosités opposées (Synnott, 1987). Les traitements de la pilosité ainsi que leurs emplacements sont genrés et idéologiques, ce qui donne aux poils «une richesse et puissance particulière, peut-être unique, en tant que symbole public et physique de l'individu [soi] ; dans les trois zones et

${ }^{2}$ Les rastafaris optent pour les dreadlocks.

${ }^{3}$ Le Mouvement nappy ou le mouvement du retour au cheveu naturel. Le mot anglais nappy signifie notamment «crépu». 
dans les quatre modes de changement de cheveux, les normes pour les hommes et les femmes sont opposées» (Synnott, 1987, p. 382-383). La pratique du défrisage illustre cette théorie : les poils de la tête sont défrisés contrairement aux autres poils. Cependant, l'homme et la femme ont une pratique différente du défrisage, les idéologies différentes (rastafari, nappy etc.) considèrent différemment le défrisage.

\section{Méthodologie}

M'intéressant dans le cadre de ma recherche doctorale aux traitements de la chevelure crépue dans les processus de socialisation et d'intégration, j'ai réalisé trois ethnographies, dont une sur la pratique du défrisage entre 2011 et 2014. J'ai mené une grande partie de l'enquête en France, à Strasbourg et à Marseille où je séjournais chez une de mes sœurs à Noailles, quartier africain aux nombreux salons de coiffure et boutiques africaines ${ }^{4}$. J'ai effectué deux séjours au Cameroun, à Yaoundé et à Douala. Le premier en août 2012, durant deux semaines, le second en juin-juillet 2014. Dans les deux cas, je résidais chez des membres de ma famille et vivais en immersion complète.

J'ai appliqué des méthodes qualitatives et quantitatives. Les données qualitatives proviennent de carnets de terrains, de 43 entretiens semidirectifs (37 femmes et 6 hommes de 19 à 57 ans) et d'entretiens informels ; d'observations participantes et flottantes (Pétonnet, 1982); de photographies des lieux, des coiffures, des séances de coiffure, du matériel et des produits capillaires, etc. ; de vidéos d'entretiens et d'observations.

Pour les données quantitatives, j'ai élaboré avec le logiciel Sphinx@ un questionnaire anonyme ${ }^{5}$ sur le traitement des cheveux défrisés et les représentations en rapport avec le cheveu crépu. 152 personnes y ont répondu : 111 ayant entre 17 à 48 ans au Cameroun et 41 en France entre 18 et 46 ans. Ce sont majoritairement des jeunes et des femmes. Bien que socialement hétérogène, cette population est relativement homogène,

${ }^{4} J^{\prime}$ utilise également les données quantitatives et qualitatives d'une ethnographie des cheveux crépus que j'ai menée en France (Strasbourg, Lyon, Marseille) en 2010-2011.

${ }^{5}$ Sauf indication contraire, les prénoms sont changés. Certains informateurs ont néanmoins insisté pour que leur nom ainsi que leur visage apparaissent. 
environ deux tiers des répondants sont Camerounais ou originaires du Cameroun, un tiers est originaire d'autres pays subsahariens et de la France d'outre-mer.

Je suis née au Cameroun et j'ai, comme mes répondants, des cheveux crépus qu'il m'est arrivé de défriser. Pour cette recherche, j'ai adopté une démarche émique (de Sardan, 1998) afin de comprendre de l'intérieur le vécu de mes informateurs, de voir à travers leurs points de vue. Partant de leurs expériences, j'ai réfléchi à partir de leurs discours et de leurs catégories, ce qui m’a permis de confronter leurs réalités et les recherches antérieures. Je fonde ma réflexion sur mes données de terrain et les travaux de mes prédécesseurs, en particulier la théorie des contraires ou des opposés (Synnott, 1987). Ces trois oppositions définies par l'auteur - corps/tête, homme/femme, idéologies - permettent de concevoir conjointement les pratiques et les représentations, mais également d'extirper les cheveux crépus de leur ghetto ethnique pour les intégrer aux autres types de chevelure. L'individu et la société, à travers les idéologies articulées avec les oppositions, façonnent des identités normées, imaginaires, mais opérantes.

\section{Le défrisage, pratique mixte}

À travers l'examen et l'analyse des pratiques capillaires des populations noires, en particulier le défrisage, les auteurs questionnent et définissent I'identité noire (Thompson, 2008-2009 ; Lester, 1999 ; Patton, 2006). Il y a une sorte de crispation sur cette pratique capillaire qui cristallise la honte, la colère, la négation et le rejet de la condition noire pour certains, en particulier dans les territoires post-esclavagistes. Les questions identitaires coagulent autour de ce phénomène (comme de la dépigmentation volontaire) depuis sa remise en cause dans les années 1950-1960 par les Afro-américains.

Contre toute évidence ${ }^{6}$, le défrisage apparaît comme une pratique féminine. Les auteurs omettent habituellement les hommes des enquêtes et travaux (Sméralda, 2004 ; 2012 ; Patton, 2006 ; Eyene, 2008 ; Thomas, 2013). Les travaux abordent le cheveu crépu ou défrisé, mais se focalisent

${ }^{6}$ Aux Antilles, les hommes défriseraient rarement leurs cheveux, cette pratique étant assimilée à une féminisation (entretien informel avec Corinne Mencé-Caster, Symposium franco-japonais, Strasbourg, 26 septembre 2019). 
sur les femmes noires. Les hommes sont absents ou minorés dans ce questionnement depuis la fin du Mouvement de Lutte pour les Droits civiques (années 1950-1960), comme s'ils avaient renoncé au défrisage. Le défrisage masculin est occulté tandis que le féminin est critiqué, en particulier par les nappys. Le Mouvement de retour au cheveu naturel ou Mouvement nappy rejette le défrisage et exhorte les femmes crépues, pas les hommes, à revenir au cheveu naturel. Il tente de renverser le stigmate qui touche les femmes noires aux cheveux crépus en affirmant leur beauté et leur féminité (Sméralda, 2004 ; Wilkerson, 2017).

Il est difficile d'identifier les hommes noirs défrisés (Photo 1). Comme d'autres auteurs, je les ai un peu négligés au départ. Pourtant, les observations dans les salons de coiffure en France comme au Cameroun pointent la mixité et la régularité du défrisage. En France, seulement trois hommes ont répondu au questionnaire sur le défrisage. Deux indiquent qu'ils ont un «dégradé avec une crête» (Photo 2), le troisième a un «dégradé» (Photo 1). Dans les trois cas, la nuque et les oreilles sont dégagées. Les données collectées au Cameroun ainsi que de nombreuses observations dans les salons de coiffure africains en France comme au Cameroun compensent le peu de répondants du terrain français et confirment la mixité du défrisage.

À Strasbourg, j'ai eu la chance de travailler avec Kevin qui a ouvert peu après le début de ma recherche doctorale un salon de coiffure masculin alors que je travaillais sur les coiffures des femmes ${ }^{7}$. J'y ai observé une clientèle majoritairement masculine, contrairement au salon mixte dans lequel il travaillait auparavant. II y a bien plus d'hommes aux cheveux défrisés que l'observation directe ou les publications ne le laissaient présager. À l'exception de jeunes hommes arborant crête et coupe punk comme leurs idoles, souvent des footballeurs comme Neymar et Balotelli, rares sont les hommes noirs aux cheveux visiblement défrisés. Ils défrisent des cheveux courts, voire très courts (Photo 1). C'est ainsi qu'ils passent inaperçus. Une fois cela observé et compris, j'ai facilement repéré les hommes défrisés au Cameroun.

${ }^{7} C^{\prime}$ est la raison pour laquelle je ne dispose que de données détaillées pour trois hommes en France. 
Photo 1 : Ludovic coiffe son collègue $\mathrm{Ali}^{8}$, défrisé la veille, Tropicoiff, 28 octobre 2010

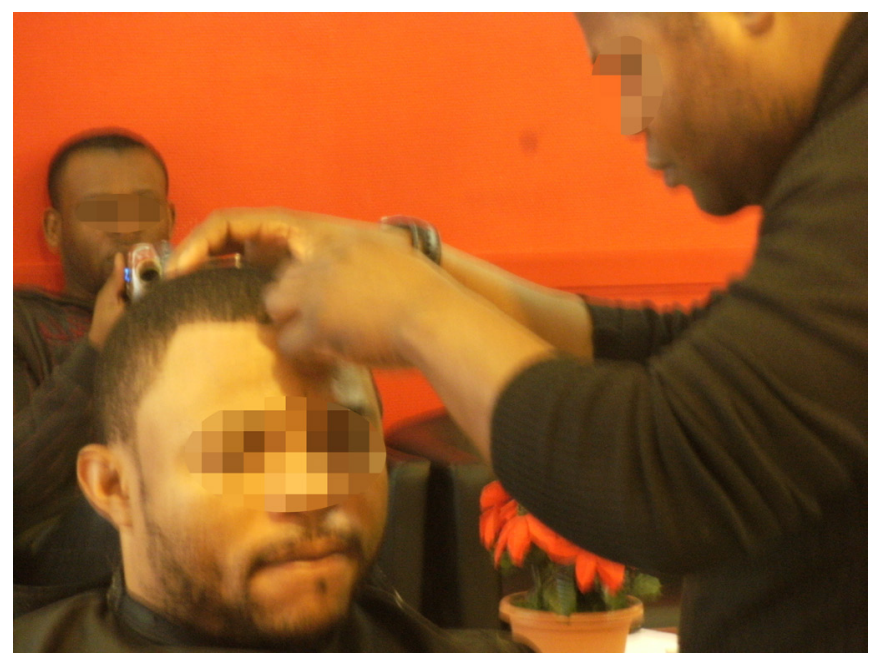

Photo 2 : Crête défrisée, Salami via Facebook@, Strasbourg, 2 juin 2015

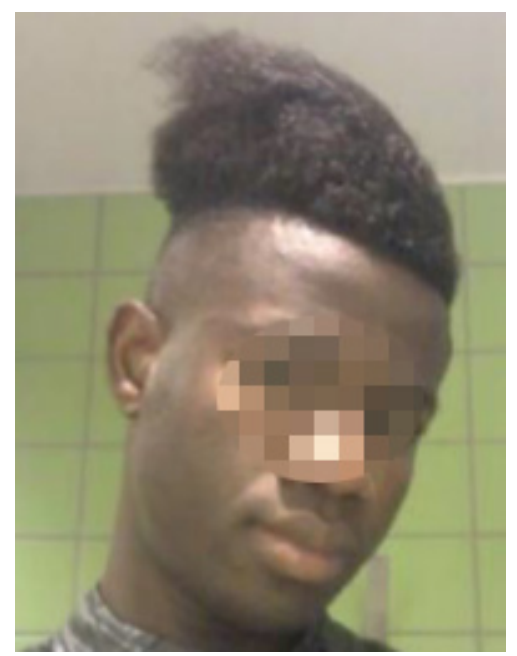

${ }^{8}$ Vrais prénoms.

Sociologias, Porto Alegre, ano 21, n. 52, set-dez 2019, p. 74-103. 
Dans ce pays, ils ont en majorité (43 sur 56) des cheveux relativement longs (plus de dix centimètres), souvent redressés (crête ou coupe punk). Le contexte «footballistique » et la jeunesse des hommes consultés expliquent la fréquence de ce type de coiffures. Une douzaine d'hommes au crâne rasé et un seul a une coupe courte, cependant l'observation tend à montrer que les hommes défrisés ont plus souvent les cheveux courts que long.

Photo 3 : Rinçage du produit défrisant, Tropicoiff, Strasbourg, 16 octobre 2010

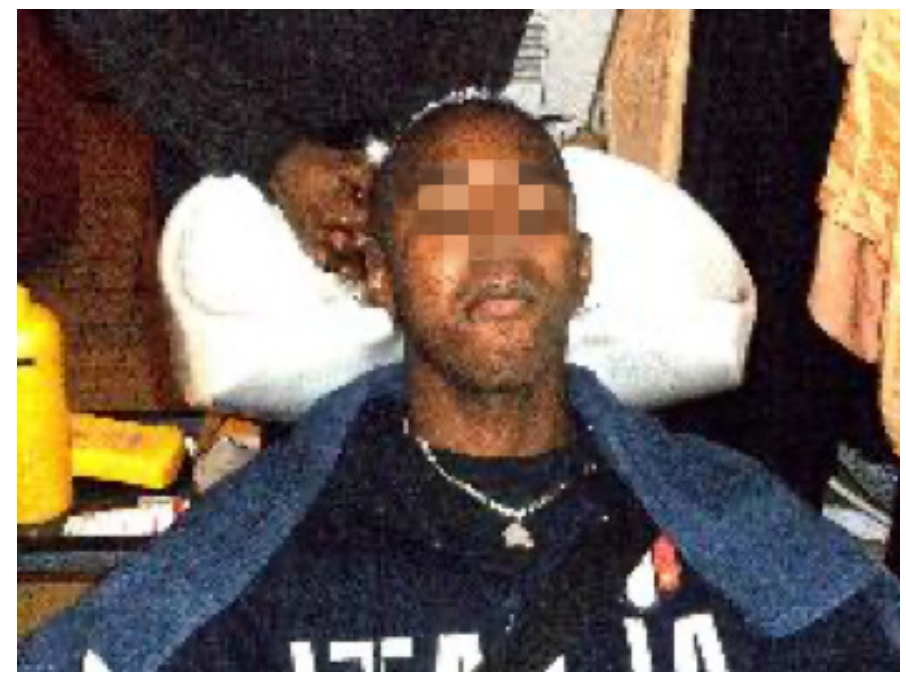

De leur côté, les femmes ont tendance à garder des cheveux relativement longs. Du fait de la longueur des cheveux et des coiffures choisies (tissage, nattes et tresses avec mèches), le défrisage féminin est souvent évident, contrairement au masculin. Certaines coiffures masquent quelque peu le défrisage. C'est la longueur qui révèle le défrisage. Or, la longueur est associée au féminin, en opposition avec le masculin, de façon symbolique. Cheveux longs $=$ féminin. Cheveux courts = masculin. Sa mixité établie, je comparai différents aspects du défrisage afin de déterminer dans quelle mesure cette pratique diffère selon le sexe. 


\section{Rituel de passage versus tendance}

En France, l'âge pour le premier défrisage varie entre 3 ans et 37 ans. Pour les trois hommes interrogés, c'est respectivement 15 ans, 18 ans et 25 ans. La situation diffère pour les femmes. La puberté est la période pendant laquelle la majorité des femmes expérimente leur premier défrisage. Ainsi, presque 9 femmes sur 10 commencent le défrisage après 10 ans, dont près de deux tiers après leurs 14 ans.

Ouh, on va dire que ça a commencé genre euh, à l'adolescence, ça veut dire euh peut-être sixième [...] Sixième, donc, 12, 13 ans (Danielle ${ }^{9}, 26$ ans, Strasbourg).

Ben, je pense que le premier défrisage j'ai dû le faire quand j'avais euh quand j'avais, ouais, peut-être 12,13 ans [...] donc j'en ai trente(Vénus, 30 ans, Lyon).

Ouh, oh, attends. 15 ans! (Paola, 30 ans, Strasbourg).

Par ailleurs, moins d'un tiers des femmes commence le défrisage après ses 20 ans. C'est le cas de Victoire, 28 ans, qui a ses cheveux défrisés depuis l'âge de 23 ans. Par contre, peu de femmes expérimentent le défrisage avant 10 ans, comme Nadège qui à 38 ans a les cheveux défrisés pour la première fois à 8 ans. Il est assez rare de défriser les filles (et les garçons) avant leur entrée au collège, en somme avant la puberté. Au Cameroun, I'âge du premier défrisage varie entre 5 ans et 43 ans. Peu de femmes expérimentent le défrisage avant leurs 10 ans comme Mami qui a 23 ans et est défrisée depuis ses 8 ans.

Au Cameroun, presque toutes les femmes commencent après 14 ans. Pour environ deux tiers d'entre elles, c'est entre 14 et 20 ans. C'est de cas de Lydia qui commence le défrisage vers 14 ans. Une dizaine de femmes font l'expérience du défrisage après la vingtaine, comme Véronique ${ }^{10}$ qui s'y met à 40 ans : «Moi, je me défrise, ça fait trois mois, même six mois même, que je mets le pot. [...] Parce que c'est souvent difficile à peigner!» (Véronique, 40 ans, Yaoundé). S'agissant des hommes, Igor, un étudiant de

${ }^{9}$ Vrai prénom.

${ }^{10}$ Vrai prénom. 
23 ans est le seul à expérimenter le défrisage avant ses 10 ans. La majorité des hommes commence le défrisage après ses 14 ans, dont la moitié après I'âge de 20 ans, par exemple Serge (commerçant, 30 ans, Yaoundé) qui commence à 29 ans.

Sur nos deux terrains, les femmes commencent généralement le défrisage avant les hommes. Contrairement à ces derniers, leur premier défrisage est souvent en relation avec leur puberté. Les hommes ont plutôt tendance à tester le défrisage sur une période qui s'étale de l'adolescence à l'âge adulte, sans relation particulière avec leur puberté. Il s'agit là d'une différence importante quant à la fonction du défrisage. D'un côté, le premier défrisage représente un rituel de passage pour les filles et adolescentes (Herron, 1998, Sméralda, 2012), une entrée dans l'univers des femmes. De l'autre, il s'agit d'un moment parmi d'autres pour les hommes.

\section{Une pratique durable}

Le défrisage a pour effet de détendre les cheveux, mais les repousses sont crépues, ce qui nécessite un renouvellement du traitement après un certain temps. Il s'inscrit alors dans la durée, en particulier pour les femmes qui gardent souvent leurs cheveux longs, contrairement aux hommes. Comme elles commencent souvent le défrisage plus tôt que les hommes, la durée depuis laquelle elles se défrisent devrait être plus longue que pour les hommes. Qu'en est-il ?

En France, la durée depuis laquelle les personnes défrisent leurs cheveux varie de moins de 1 an à 30 ans. Environ un quart des femmes défrise ses cheveux depuis moins de 5 ans au moment de l'enquête de terrain, comme Marinella et Netty qui se défrisent alors depuis un an. À I'opposé, trois quarts des femmes se défrisent depuis au moins 5 ans et une moitié depuis au moins 10 ans. Nicole se défrise depuis 25 ans avec un produit défrisant pour enfant, Just for $m e{ }^{\circledR}$, trois fois par an, «quand il y a trop de repousses». Parmi les trois hommes, un homme se défrise depuis 8 ans et les deux autres depuis un an.

Au Cameroun, deux tiers des hommes et femmes consultés ont une pratique du défrisage relativement récente, tout au plus 4 ans. Pour trois quarts des hommes, le défrisage est une pratique récente. Franck, un 
étudiant se défrise depuis 2 ans et Samuel, un taximoto, depuis un an. En revanche, pour un quart d'entre eux, le défrisage s'inscrit sur une plus longue durée, au moins 5 ans. C'est le cas de Lionel, un étudiant de 21 ans qui se défrise depuis 7 ans. S'agissant des femmes, un peu plus de la moitié se défrise depuis 4 ans au maximum. Les autres ont donc une plus longue habitude du défrisage, comme Bibiche, une commerçante de 38 ans qui se défrise depuis 18 ans.

En France, le défrisage s'inscrit dans une durée relativement longue, plus de 5 ans tandis qu'au Cameroun, pour la majorité des répondants, c'est une pratique assez récente, tout au plus 4 ans. L'une des raisons à cet écart est que dans la plupart des établissements scolaires camerounais, les garçons comme les filles doivent se conformer à un règlement qui proscrit la majorité des traitements capillaires, dont le défrisage. Dans ces conditions, pendant les périodes scolaires, les élèves des deux sexes doivent respecter des règles capillaires. Crâne rasé ou tondu pour les garçons, quelques tresses plaquées pour les filles. Il y a là à la fois une différence entre hommes et femmes, mais aussi entre terrain français et camerounais. Qu'en est-il de la fréquence du traitement?

\section{Fréquence}

Le défrisage nécessite de la régularité. Sur les notices, il est recommandé de défriser les repousses crépues environ toutes les 6 semaines. Certains comme Vénus suivent les instructions: «Mais si je porte les cheveux sans les tresses, je les défrise, on va dire toutes les 6, 7 semaines». Mais au Cameroun comme en France, soit, elles espacent trop les défrisages :

Je défrise tous les trois mois [...] Mais actuellement, ça fait quatre mois, je me suis dit qu'il fallait laisser reposer le cuir chevelu [...] Et puis avec la saison où il fait froid, je me suis fait des rastas (Cécile ${ }^{11}, 45$ ans, Yaoundé).

Je me fais défriser [...] une fois tous les six mois [...]. Euh, je n'ai pas les cheveux crépus [...] Frisés, oui (Leslie, 30 ans, Strasbourg).

${ }^{11}$ Vrai prénom. 
Un mois, je fais les tresses, un mois, je les enlève, j'attends deux semaines, après je les défrise (Armand ${ }^{12}, 30$ ans, Strasbourg).

Ou alors, elles rapprochent trop les défrisages, comme Nanou, assistante maternelle de 35 ans pour qui c'est «toutes les deux semaines». Dans l'ensemble, les défrisés consultés ne respectent donc pas vraiment les recommandations des fabricants des produits utilisés. Les hommes ont tendance à renouveler leur défrisage plus souvent que les femmes. Par exemple, Serge défrise ses cheveux toutes les semaines. En discutant avec Kevin, coiffeur à Strasbourg, je comprends qu'ils procèdent souvent comme lui : il tond la partie défrisée et défrise les repousses.

Les défrisages trop fréquents ou trop espacés abîment les cheveux. Trop fréquents, ils les altèrent et provoquent leur chute et/ou la brûlure du cuir chevelu. Trop espacés, ils les fragilisent : la différence de texture entre les parties crépue et défrisée fragilise la chevelure et entraîne sa casse, en particulier lors du peignage et du brossage. D'où la nécessité de respecter les instructions. Les coiffeurs professionnels devraient respecter ces recommandations, mais ils suivent souvent le désir des clients, ou alors ils insistent sur la nécessité d'un défrisage. Les cheveux défrisés seraient plus faciles à coiffer que ceux crépus. Il est possible de défriser ses cheveux soi-même ou de faire appel à une tierce personne. Se pose alors la question du coiffeur. Qui s'occupe de ce traitement qui comporte tout de même des risques?

\section{Le coiffeur}

En France, parmi les 3 hommes consultés, I'un se défrise seul, un autre paie un coiffeur et le dernier fait appel à une relation. Les observations de terrain vont dans le même sens. Rares sont les hommes qui se défrisent seuls. Ils confient plutôt cette tâche à un tiers, souvent une femme de leur entourage ou leur coiffeur. Contrairement aux hommes, la moitié des femmes se défrise elle-même : «Je le fais moi-même [...] Je le fais pour économiser de l'argent [...] Puisque je sais le faire, pourquoi aller chez le

\footnotetext{
${ }^{12}$ Vrai prénom.
} 
coiffeur?» (Leslie, 30 ans, Strasbourg). L'autre moitié le confie à une tierce personne, habituellement une femme de son entourage :

Je crois que c'est maman qui m'a défrisé plusieurs fois les cheveux et euh une marraine, ma marraine, ma tante (Danielle, 26 ans, Strasbourg).

Là, il y a ma mère qui m’a défrisée pour la première fois, parce que ma mère n'est pas du tout patiente pour la coiffure (Nell, 19 ans, Strasbourg).

D'autres privilégient un coiffeur professionnel. C'est moins fréquent et souvent en lien avec une autre coiffure comme la pose d'un tissage (Photo 4) ou des soins après-défrisage :

Photo 4 : Charlène $^{13}$, défrisage et tissage partiel, Yaoundé, 24 juin 2014

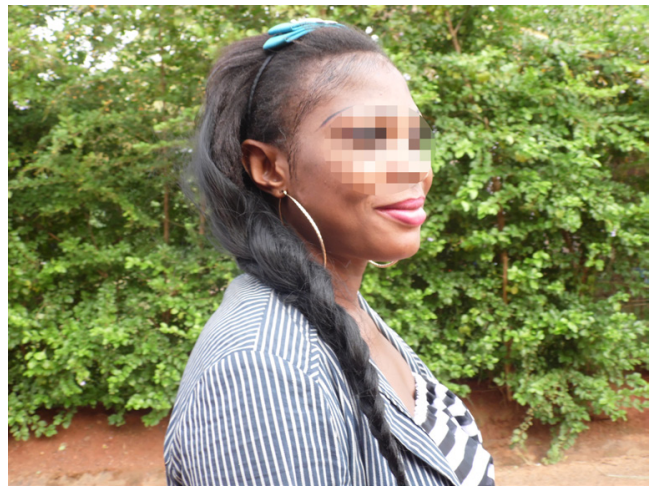

Et donc en général, ici à Lyon quand je vais au salon de coiffure, c'était chez Ivoire et ébène et ils utilisent Revlon ${ }^{\circledR}$ (Vénus, 30 ans, Lyon).

En salon, parce qu'après le défrisage, il faut que je fasse une mise en plis, donc qui dit rouleaux, bigoudis, rentrer sous le casque et après tirer, seule j'arrive pas à le faire (Paola, 30 ans, Strasbourg).

\footnotetext{
${ }^{13}$ Vrai prénom.
} 
Au Cameroun, la situation diffère. D'une part, la majorité des hommes comme des femmes confie son défrisage à un tiers, habituellement le coiffeur. L'entourage familial et amical intervient parfois, mais moins souvent qu'en France. Une infime partie des hommes et des femmes défrisés se charge de son propre défrisage. La visite chez le coiffeur ou la coiffeuse (professionnel ou amateur) est donc une activité régulière, voire obligatoire comme pour les enfants scolarisés. De plus, les coiffeurs et les produits défrisant sont à des tarifs plus accessibles au Cameroun qu'en France, d'où une plus importante fréquentation des salons de coiffure et des coiffeurs informels (Photo 5, Photo 6, Photo 7, Photo 8).

Photo 5 : Iris ${ }^{14}$ tressée à Mvog Ada, entre deux maisons, Yaoundé,

11 juin 2014

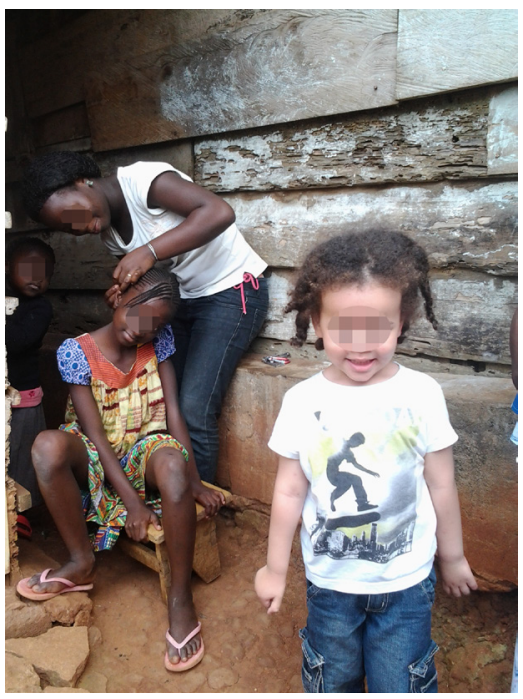

Photo 6 : Rachel tresse une cliente défrisée dans son salon, Yaoundé, 3 juillet 2014

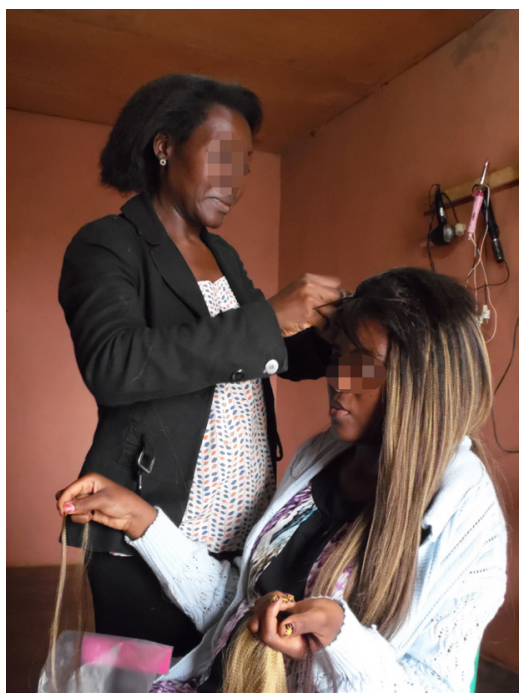

${ }^{14}$ Vrais prénoms pour Iris, Rachel et Anna. 
Photo 7 : Une voisine coiffe les cheveux défrisés d'Iris chez sa grand-tante Anna, dans la cour, Douala, 18 juin 2014

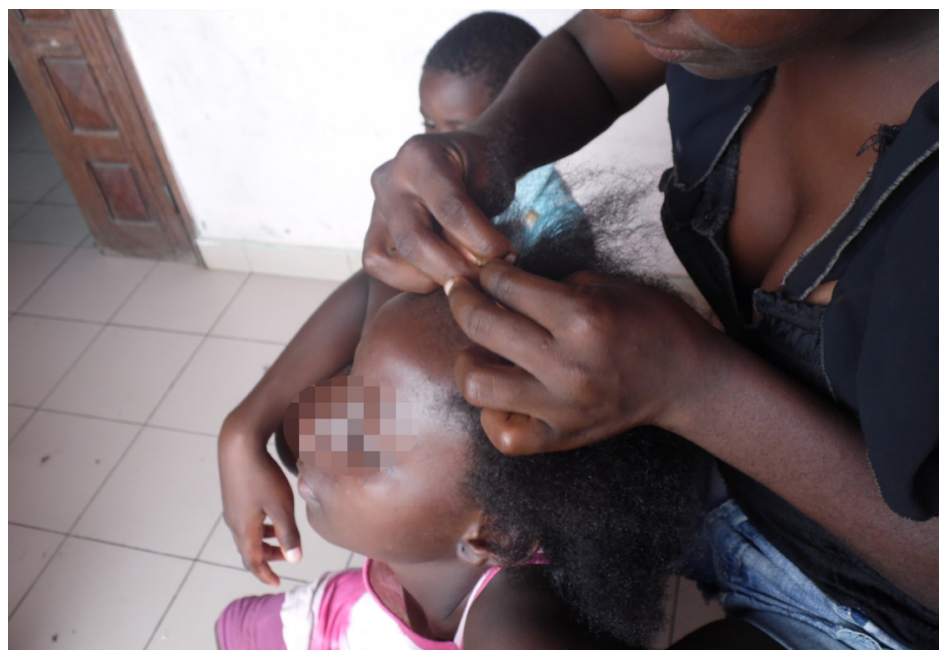

Photo 8 : Iris, tresses renversées, Douala, 18 juin 2014

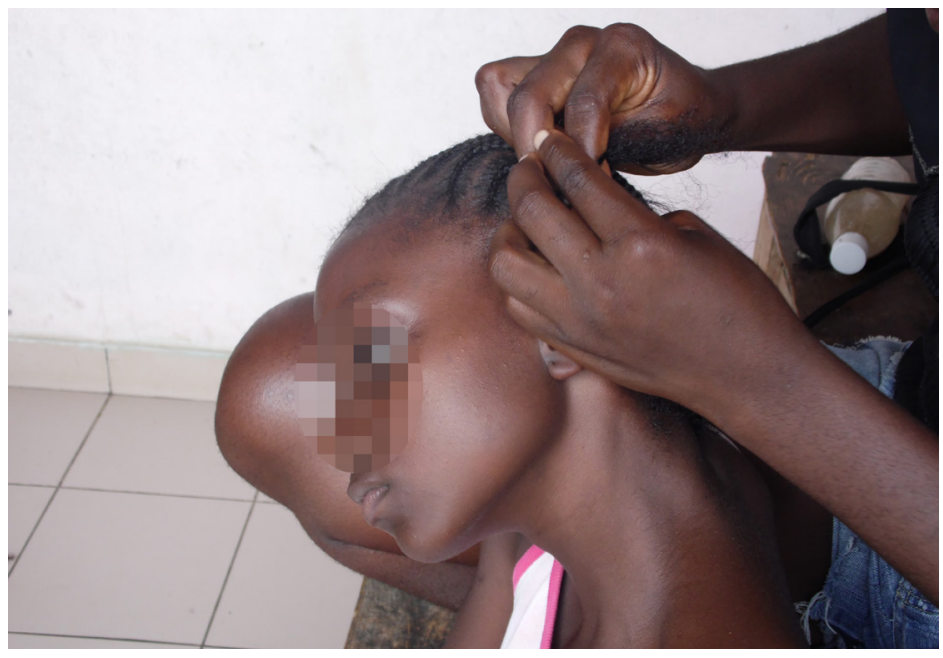

Sociologias, Porto Alegre, ano 21, n. 52, set-dez 2019, p. 74-103. 


\section{Entre soucis et rejet}

«Mais en fait, ils sont cassés»

Comme d'autres produits chimiques, les crèmes relaxantes ont des contre-indications et des effets indésirables. Le défrisage fragilise la chevelure et l'assèche ainsi que le cuir chevelu. La manipulation d'une crème relaxante nécessite donc quelques précautions parmi lesquels : effectuer un essai sur une petite partie de la chevelure 24 heures avant, ne pas utiliser de matériel métallique, ne pas associer avec du henné, etc. À défaut, le défrisage peut s'avérer désastreux. «Bien sûr! Un défrisage permanent, ça casse, ça casse le cheveu» (Paola, 30 ans, Strasbourg).

En France, un tiers des répondants a connu un souci après un défrisage, généralement des brûlures des cheveux ou du cuir chevelu, des chutes ou des cheveux cassés :

Parce que j'ai constaté que les défrisants, c'est bien beau quand on les défrise, mais après ça casse le cheveu [...] Parce que ces défrisants, quand même, ça brûle la peau! (Erica, 39 ans, Strasbourg).

Déjà cramé le crâne, le cuir chevelu, des croûtes, ça brûlait quand je lavais les cheveux. Après, avec les croûtes, ça a fait des pellicules (Gigi, 25 ans, Strasbourg).

Cuir chevelu fragile, ça brûle même avec le produit pour bébé. À chaque fois, cuir chevelu fragile (Ramona, 45 ans, Strasbourg).

Brûlé les cheveux par une coiffeuse, c'est pour cela que je fais moi-même. Il y a 4-5 ans (Mandy, 40 ans, Strasbourg).

Après, quelques brûlures, la tête devenue sensible à cet endroit (Annabella, 26 ans, Strasbourg).

Mais en fait, ils sont cassés (Soraya, 19 ans, Strasbourg). 
La situation est comparable au Cameroun. Un tiers des répondants, hommes comme femmes, a connu un incident à la suite d'un défrisage, avec les mêmes conséquences :

La perte des cheveux (Jamar, 27 ans, Yaoundé).

Les cheveux se sont coupés (Fabien, 20 ans, Yaoundé).

Mes cheveux sont devenus fragiles (Gabriel, 25 ans, Yaoundé).

Les cheveux deviennent trop tendres et tombent facilement(Léona, 18 ans, Yaoundé).

Ma peau s'est brûlée, ce qui m'a rendu un peu affreux (Forrest, 20 ans, Yaoundé).

Contre toute attente, bien que le défrisage soit dans la majorité des cas réalisé par un tiers, c'est dans une même proportion qu'en France que les défrisés connaissent des dommages relatifs au défrisage. Ces ratés sont souvent la cause directe de l'arrêt de cette pratique, au-delà de toute autre considération. Mais pour beaucoup, il est difficile d'arrêter :

Et quand je les défrise, en fait le truc c'est que, ben, je ne m'en occupe pas bien. Au moment de dormir, je sais pas, j'aime pas mettre quelque chose sur ma tête pour dormir donc du coup ça frotte, ils s'abîment, ou alors quand j'ai des repousses, je fais pas le défrisage tout de suite donc ça se casse. Donc, en fait quand je les défrise, hum, j'ai l'impression qu'à long terme, sur le long terme je les abîme parce que je les entretiens pas bien. Et, et si je les défrise pas, euh, j'arrive pas à les coiffer. Mais idéalement, j'aimerais bien pouvoir avoir euh, ouais les cheveux au naturel, mais que j'arrive à coiffer comme je veux quoi (Vénus, 30 ans, Strasbourg).

Malgré les risques, malgré les tentatives pour renoncer au défrisage et garder les cheveux «naturels», cette pratique demeure une pratique fréquente, dans les salons comme ailleurs. Elle semble incontournable. 
«On le défrise.»

Tableau 1 : Avis sur le cheveu crépu (France et Cameroun)

\begin{tabular}{|l|c|c|c|}
\hline Que pensez-vous du cheveu crépu? & Femme & Homme & TOTAL \\
\hline C'est bien, j'aime bien & 20 & 25 & 45 \\
\hline Difficile à entretenir, à coiffer & 28 & 4 & 32 \\
\hline C'est moche, horrible, répugnant, dégoûtant, laid & 16 & 10 & 26 \\
\hline C'est beau, joli & 9 & 8 & 17 \\
\hline C'est pas bien & 10 & 7 & 17 \\
\hline Sans avis & 7 & 0 & 7 \\
\hline C'est sale & 2 & 5 & 7 \\
\hline Réponse non valide & 1 & 0 & 1 \\
\hline TOTAL & $\mathbf{9 3}$ & $\mathbf{5 9}$ & $\mathbf{1 5 2}$ \\
\hline
\end{tabular}

En France comme au Cameroun, un tiers des enquêtés a connu un effet indésirable après un défrisage : casse, chute, brûlure. Malgré tout, la plupart continuent de défriser leur chevelure crépue. La stigmatisation et la dévalorisation des cheveux crépus à la suite de l'esclavage et de la colonisation en seraient les causes (Sméralda, 2004 ; 2012). Cependant, dans quelle mesure les personnes concernées, celles qui se défrisent, déprécientelles leurs cheveux crépus? La dévalorisation des cheveux crépus est-elle systématique, communément admise par tous les défrisés? Si certains n'aiment effectivement pas leur chevelure crépue, tous ne partagent pas cette opinion comme l'indique l'ethnographie.

En France, 35 répondants sur les 41 consultés expriment une opinion sur les cheveux crépus. Elles sont réparties dans quatre catégories, par ordre décroissant : «difficile, dur à entretenir, à coiffer», «bien, très bien», «pas bien», et «beau, joli». Près de la moitié des personnes défrisées pense que le cheveu crépu est «difficile à entretenir, à coiffer». D'une part, l'accent est mis sur la difficulté à coiffer les cheveux crépus. Pour Marinella, «c'est une bonne chose, sauf qu'on peut pas faire toutes sortes de coiffure avec». Pour 
Brian, les «cheveux ne gênent pas quand il y a le vent», mais il éprouve de la «difficulté pour faire une coiffure». Dina est du même avis, le cheveu crépu est «trop dur à coiffer», même s'il est «intéressant».

Ainsi, des femmes utilisent le défrisage pour démêler leurs cheveux jugés trop durs à peigner. Elles les «soufflent», ou «assouplissent». Ce dernier terme met l'accent sur la supposée dureté des cheveux crépus, reprenant ainsi la terminologie des marques de produits défrisant. Les cheveux crépus seraient durs, il faut les adoucir/assouplir pour pourvoir les coiffer. Lorsque je demande à Erica si ses cheveux sont défrisés, je ne m'attends pas à ce qu'elle me réponde par la négative, car pour moi, ils sont visiblement défrisés et je l'ai déjà vu faire :

Il est pas défrisé, il est soufflé [...] Soufflé, c'est-à-dire que, que moi je peigne de temps en temps avec un peigne chaud, euh, pas très chaud! [...] Donc, pour, pour euh pour le démêler [...] Parce qu'il est tout le temps touffu [...] Et ça fait que ça fait mal quand on peigne (Erica, 39 ans, Strasbourg).

D’autre part, les défrisés insistent sur la difficulté à entretenir une chevelure crépue, justifiant ainsi le défrisage :

Je trouve qu'il est très dur à entretenir. C'est bien le retour au naturel», mais, le cheveu crépu fait vite négligé, quand on entretient pas. C'est pour cela qu'on est obligé de faire des coiffures protectives (Maya, 26 ans, Strasbourg).

Dans une moindre mesure, quelques répondants du terrain pensent que le cheveu crépu est «bien, très bien». Florence précise : «Je reviens au crépu tous les 2 ans. J'aime bien pour changer». Le défrisage n'est pas particulièrement le fait de personnes qui détestent leurs cheveux crépus ou qui en ont honte. De fait, une faible proportion des répondants en France pense que le cheveu crépu n'est «pas bien». Ainsi, Nicoletta s'exclame : «Oh! Les cheveux crépus, c'est pas du tout bien, pas du tout bien. C'est pas présentable. Pas du tout.» Là encore, un aspect important est souligné. Pour elle, le cheveu crépu ne serait pas «présentable». II serait désordonné, au contraire du cheveu caucasien, inévitablement ordonné, symbole de l'ordre comme du beau. 
À Strasbourg, Nicole me précise : «c'est pas bon. Quand les cheveux sont lisses, c'est bon, c'est facile à coiffer, c'est pour ça qu'on met le défrisant». Nerys a un avis catégorique : le cheveu crépu «on le défrise». À I'opposé, seulement deux répondantes, Mariama et Marianne, pensent que le cheveu crépu est «beau», «joli». Marianne trouve aussi que «c'est beau!» Néanmoins, elle ajoute que le défrisage «c'est bien pour les cheveux, pour les rendre mieux même si on perd de temps en temps quelques-uns». Cela illustre bien la position de beaucoup qui estiment que le défrisage améliore les cheveux crépus malgré les dégâts occasionnels. Or, un tiers des répondants a connu un épisode «douloureux» à la suite d'un défrisage : casse, chute, brûlures. Malgré cela, les personnes continuent à défriser leur chevelure même lorsque celle-ci est visiblement abîmée.

En répartissant les différentes opinions formulées dans deux catégories, «image positive» et «image négative» du cheveu crépu, je constate qu'en France environ un quart des répondants a du cheveu crépu une «image positive». La majorité, six répondants sur dix, en ont une «image négative». En mettant de côté les trois hommes, car leur nombre n'est pas significatif, ce sont donc des femmes qui ont tendance à avoir une opinion négative des cheveux crépus. Il serait difficile à entretenir et à coiffer.

Cette opinion est moins répandue au Cameroun où deux nouvelles catégories surgissent des données : «moche, horrible...» et «sale». Un tiers des répondants camerounais pense que le cheveu crépu est «bien». C'est le cas de Flora une étudiante de 18 ans. Pour Darren, le cheveu crépu, c'est «génial». Moins d'un quart des répondants pense que le cheveu crépu est «moche, horrible». Ils expriment là leur répugnance. Comme Serge pour lequel le cheveu crépu est « répugnant ». De leur côté, Giovanni, Nanie et Fatimatou (25 ans) pensent qu'il est «moche». Magelan, médecin de 30 ans, estime qu'il est «affreux». Dans le même ordre d'idée, Marcello le trouve «effrayant». Pour Dexter, le cheveu crépu, «c'est horrible».

Au contraire, pour une petite partie de répondants, le cheveu crépu est «beau, joli». Pour Ojim, il est «beau», tout comme pour Ferdinand qui le trouve «beau à voir». Quelques répondants seulement pensent que le cheveu crépu est «difficile à entretenir, à coiffer». Pour Harry, le cheveu crépu, «c'est beaucoup d'entretien». Pour Zoé, «c'est trop dur». Silvane 
précise qu'il «faut tout le temps les traiter». Pour Magnolia, «c'est pénible à coiffer». Samira et Emmanuel pensent que le cheveu crépu n'est «pas aisé à coiffer».

La coiffure est toujours là! Or, le cheveu naturel, tu ne peux pas peigner ça! [...] Tu ne tresses pas! Tu ne coupes pas. Parce que c'est souvent difficile à peigner ! [...] Difficile à peigner! Tu peignes, tu pars [...] au travail avec, demain tu es obligée de changer la coiffure [...] Moi, je me défrise, ça fait trois mois, même six mois même, que je mets le pot [...] Parce que c'est souvent difficile à peigner! [...] Mais je peux me défriser, je garde ça, ça met un, deux mois, c'est toujours là! Je ne mets pas de greffe [...] Tu défrises parce que c'est difficile à peigner! Tu ne peux pas entretenir ça sans [...] C'est pourquoi j'ai dit que, pour garder les cheveux naturels, c'est souvent difficile! (Véronique, 40 ans, Yaoundé).

Cécile ${ }^{15}$ défrise ses cheveux tous les trois mois parce que selon elle, ainsi «ils sont plus faciles à entretenir». Elle entretient son lissage dans un salon de coiffure. Pour elle, les cheveux crépus sont trop durs à entretenir dans leur forme originelle. Sa fille Inès ${ }^{16}$ partage cette opinion. Comme d'autres femmes, elle applique le défrisant sur sa chevelure pour «l'assouplir» et non pour la rendre totalement lisse. D'ailleurs, la plupart des coiffures qu'elle affectionne cachent sa chevelure. Bien que ses cheveux ne soient pas défrisés le jour de l'entretien, elle me précise qu'elle les défrise souvent parce que ses «cheveux naturels sont très durs». Même avec de la crème, le peigne, «ça ne passe pas».

Le défrisage, ça aide, bon, pour les cheveux, mais seulement c'est l'entretien. [...] C'est-à-dire que, quand on défrise les cheveux, bon, il faut les traiter, il ne faut pas les laisser sinon ça va se couper. [...] Bon, je tresse, je fais quand même des tresses [...] pour arrêter les cheveux d'abord [...] Mais après, bon, après une semaine, je tresse [...] comme si j'avais les rastas, mais... oui, les rastas [...] Il y a quand même un changement : ce n'est plus debout, ce n'est plus crépu. [...] Si c'était plus facile, vraiment, je les laisserais comme ça, parce que le défrisage, ah! Tout le temps, chaque trois mois, tu dois défriser. Non, comme ça. Je crois que je les laisserais comme ça (Inès, 19 ans).

\footnotetext{
${ }^{15}$ Vrai prénom.

${ }^{16}$ Idem.
} 
Une petite partie des enquêtés pense comme Bess que le cheveu crépu, «C'est pas bien». Pour Kilian (19 ans), «c'est pas bien du tout sans entretien». Pour Elen, le cheveu crépu, «c'est mieux quand il est défrisé». Sacha partage le même avis : «c'est bien quand il est défrisé». Le défrisage serait une aide à la coiffure et à l'entretien de la chevelure crépue.

Une minorité de défrisés pense que le cheveu crépu est «sale», comme Sonny, un ingénieur de 32 ans, pour qui il «très sale». Fabien précise : «c'est pas bien, car c'est la saleté». Bien que peu de répondants déclarent ceci dans le cadre de l'enquête par questionnaire, je remarque qu'il s'agit d'une opinion largement partagée, au Cameroun comme en France. Ils expriment ainsi l'opinion commune, chez les Africains et chez les Afrodescendants, selon laquelle les cheveux crépus sont source de désordre et de souillure. Coiffer les cheveux y compris en les défrisant en éloigne de façon provisoire la souillure et le désordre.

D'ailleurs, une des significations de nappy désigne une « couche » qui contient les excréments des bébés, comparant ainsi les cheveux crépus aux défécations. On retrouve la même idée dans l'expression « crotte de bique » qui désigne de courts cheveux crépus au Cameroun. Natter ou tresser sans mèche les cheveux crépus d'une femme semble insuffisant. II faut défriser ou ajouter des mèches pour avoir l'air «propre». Ainsi, pour Charlène (Photo 4), les femmes aux cheveux crépus simplement nattés ou tressés sont sales : «Peut-être c'est un choix! Soit alors, bon elles veulent être sales. Elles ne veulent pas s'entretenir» (Charlène, 28 ans, chanteuse, 24 juin 2014, Yaoundé).

Thérèse ${ }^{17}$ a les cheveux crépus et une coupe afro. Elle s'aime ainsi, bien que cela soit au départ une obligation, le directeur de son lycée a réclamé que les filles aient les cheveux courts comme les garçons. Cependant, elle note que «d'autres gens voient que c'est la saleté» et que cela n'est pas présentable. Son entourage lui reproche de ne pas coiffer ses cheveux crépus de façon convenable. Ils seraient trop longs. II faudrait qu'elle les natte, qu'elle fasse des tresses. Ici encore, crépu est synonyme de saleté et de désordre. Anna, la grande tante de Thérèse, explique que les cheveux crépus sont sales lorsqu'ils sont trop longs.

\footnotetext{
${ }^{17}$ Vrai prénom.
} 
Pour un garçon qui se considère, tu dois avoir les cheveux à ras! Bien taillés [...] Mais une femme africaine, c'est les cheveux crépus, mais à ras [...] pas ce genre-là. Ou tu fais les tresses avec les fils ou tu fais les nattes [...] C'est trop long. Hum, hum. Et vu que tu vois, quel que soit l'entretien, on te considère toujours que c'est sale (Anna, 60 ans, Douala).

En regroupant les réponses dans deux catégories «image positive» et «image négative», je constate que près de la moitié des répondants du terrain camerounais a une «image positive» du cheveu crépu. L'autre moitié en a une «image négative».

En prenant en compte les répondants en France et au Cameroun, près d'un tiers d'entre eux pense que le cheveu crépu est «bien». Un cinquième pense que le cheveu crépu est «difficile à entretenir, à coiffer». Un répondant sur dix pense qu'il est «beau, joli», autant que ceux qui pensent que le cheveu crépu n'est «pas bien». Dans l'ensemble, moins de la moitié des répondants a une «image positive» du cheveu crépu tandis que plus de la moitié en a une image négative.

Les femmes ayant une «image négative» du cheveu crépu pensent qu'il est «difficile à entretenir, à coiffer», qu'il n'est «pas bien». Au Cameroun, elles déclarent qu'il est «moche, horrible, etc.», qu'il est «sale». Les hommes ayant une «image négative» du cheveu crépu pensent au Cameroun qu'il est «moche, affreux, horrible, etc.» et «sale». Sur les deux terrains, il n'est «pas bien» et «difficile à entretenir, à coiffer». C'est sans doute en partie en raison du style de coiffure et de la longueur des cheveux, généralement plus longs que ceux des hommes, que les femmes pensent plus souvent qu'eux que le cheveu crépu est «difficile à entretenir, à coiffer». Elles ont aussi une image relativement plus négative des cheveux crépus que les hommes.

\section{La femme défrisée : stigmate et sexisme}

Les auteurs (Lester, 1998 ; Sméralda, 2004 ; Rosado, 2007 ; Eyene, 2008 ; Wilkerson, 2017) examinent habituellement la pratique du défrisage afin de réaliser l'analyse et la critique de l'identité noire. Cependant, leur attention se porte souvent sur les femmes noires, en particulier afroaméricaines. Ils ignorent ou minorent cette pratique chez les hommes. Or, 
nous l'avons constaté, le défrisage comme d'autres traitements capillaires est mixte. Certes, la pratique diffère quelque peu selon le sexe, mais les hommes défrisés ne sont pas une exception. Pourtant la pression sociale en matière de beauté s'exerce surtout sur les femmes (Bernuzzi de Sant'Anna, 1995 ; Le Breton, 2011), et en tant que telles, les femmes noires attirent plus l'attention des chercheurs et des militants anti-défrisage comme les nappys. On leur pardonne moins qu'aux hommes une apparence négligée :

La femme est jugée impitoyablement sur son apparence, sa séduction, sa jeunesse et ne rencontre guère de salut au-delà. Elle vaut ce que vaut son corps dans le commerce de la séduction. À la manière d'un cadeau empoisonné, il n'y a qu'un "beau sexe", mais il est limité dans le temps, et le prix est lourd à payer de ce modeste privilège (Le Breton [1990] 2011, p. 233).

Ainsi, avant d'être noires, ce sont des femmes. Elles essaient de se conformer à un idéal féminin toujours mouvant, mais occidental depuis quelques siècles. L'esclavage et la colonisation ont permis la domination par la culture occidentale et son esthétique. Dans les médias, au cinéma, dans la littérature, dans la mode et même dans le sport, la femme féminine a actuellement de longs cheveux souples, lisses (Bromberger, 2010). Selon ces critères, la chevelure idéale se situe à l'opposé du cheveu crépu.

Le défrisage permet de lisser les cheveux crépus et d'ainsi les allonger. Leur longueur réelle apparaît ainsi au grand jour. II fait ainsi d'une pierre deux coups. En cela, il répond au désir d'une chevelure (plus) longue et lisse. Avec le défrisage, les femmes noires ont la possibilité de rendre leur chevelure conforme aux critères de beauté occidentaux qu'elles ont comme d'autres relativement incorporés. Car même si la chevelure est défrisée, les coiffures qu'elles choisissent sont habituellement les mêmes que lorsque les cheveux ne sont pas défrisés. Le défrisage serait alors une solution à la difficulté d'entretenir et de coiffer les cheveux crépus.

Cependant, d'une part, les Africains noirs démêlent, peignent et coiffent aisément leurs cheveux depuis des milliers d'années. D'autre part, I'esclavage et la colonisation n'ont pas éradiqué les coiffures traditionnelles telles que les nattes portées dans certaines régions. Comment expliquer alors cet argument récurrent en faveur du défrisage qui va à l'encontre de 
l'expérience ? De plus, les dommages causés par le défrisage entament peu cet avis que même les coiffeurs partagent. Les fabricants de crèmes relaxantes, de lisseurs et autres appareils défrisant, ainsi que les producteurs de produits capillaires véhiculent le même discours. Il faut maîtriser les boucles rebelles. Rendre le démêlage plus doux. Ils s'adressent principalement à une clientèle féminine bien que des hommes emploient leurs produits. Beauté et féminité vont ainsi de pair.

Tandis que les hommes gagnent en responsabilité avec des cheveux courts, les femmes gagnent en beauté avec de longs cheveux lisses. Cette longueur dévoile la chevelure défrisée. La question du défrisage s'avère donc plus cruciale pour les femmes que les hommes. Leur chevelure défrisée est plus visible que celle des hommes, dans les rues comme dans les médias. Ce traitement inégal du défrisage par les auteurs comme par les médias et industriels de la beauté confirme l'opposition homme/femme que la théorie des contraires nous expose (Synnott, 1987). Pour l'une, il s'agit de se conformer ou non au modèle dominant, pour l'autre, il s'agit d'un style parmi d'autres Selon le sexe, la pratique et sa représentation diffèrent. Même le prix de cette prestation chez un professionnel varie en fonction de la longueur des cheveux : les femmes paient ainsi généralement deux à trois fois plus que les hommes.

\section{Conclusion}

Les hommes et les femmes ne sont pas égaux face au défrisage. Défriser ou non sa chevelure ne remet pas en cause la masculinité des premiers alors qu'elle questionne la féminité des secondes. Qui plus est, la question de I'identité en rapport avec le défrisage se pose habituellement pour les femmes noires tandis que les hommes en sont exonérés (Weitz, 2001 ; Sméralda, 2004 ; 2012 ; Chapman, 2007 ; Eyene, 2008 ; Wilkerson, 2017). L'âge du premier défrisage, la durée, la fréquence, les opinons diffèrent quelque peu d'un sexe à l'autre tandis que les dommages capillaires touchent les unes et les autres dans les mêmes proportions. En somme, le défrisage est mixte, mais sa pratique et son appréhension diffèrent selon le sexe de la personne défrisée. 
Dans les sociétés occidentales et occidentalisées, il va de soi que les cheveux longs sont féminins tandis que les cheveux courts sont masculins. Bien entendu, il s'agit d'une posture idéologique où l'homme occupe la première place. En effet, ce n'est pas le défrisage de sa chevelure qui dérange, mais sa longueur, car dans un contexte occidentalisé, les cheveux longs sont associés aujourd'hui à la féminité. La pratique du défrisage questionne I'identité noire certes, mais apparemment seulement celle des femmes. La femme noire est soumise à une double injonction. La première injonction, être authentique en gardant ses cheveux crépus comme le prônent les nappys, est à l'opposé de la seconde, être belle, ce qui implique pour beaucoup le défrisage afin de conformer sa chevelure à celle des femmes non noires, notamment blanches.

La beauté a aujourd'hui les traits de l'Occidentale, à défaut de la métisse mais certainement pas de l'Africaine. La femme noire aux cheveux crépus est la plus éloignée des standards actuels de beauté. Sa peau ? Pas assez claire. Ses cheveux ? pas assez lisses. Prise entre racisme et sexisme, la femme qui défrise ses cheveux tente par là non seulement de correspondre aux standards de beauté et de réussite, mais aussi de renverser le stigmate lié à sa chevelure crépue. La société lui demande d'endosser une identité fictive, discriminante, mais opérante.

D'un point de vue sociologique, avec le défrisage, nous abordons les études noires, les black studies ${ }^{18}$ développées à la fin des années 1960, à la suite du Mouvement pour les Droits civiques. II met en lumière le rapport inégalitaire entre Blancs et Noirs. Cette pratique résulte du traitement inégal des populations noires et afrodescendantes aux USA. Le défrisage vise à rendre conforme la chevelure crépue à un idéal de beauté capillaire occidental. II met ainsi en lumière la malléabilité du corps, ici les poils, sa relative plasticité. En tant que technique du corps, le défrisage est à la fois le produit et l'instrument d'une culture particulière, ici afro-américaine. Il exprime cette culture spécifique, avec son vécu, ses traumatismes, ses stratégies. Il montre, dans le sens visuel, la condition noire dans cette culture spécifique. Dans le même mouvement, il renseigne sur l'interdépendance entre identité noire et blanche, à la suite de l'esclavage et du colonialisme,

${ }^{18}$ Voir à ce propos Caroline Rolland-Diamond (2012). 
voire du néocolonialisme. Du point de vue des Afrodescendants, les conditions noires et blanches sont inextricablement liées.

Le défrisage participe aujourd'hui d'une identité noire qui refuse le stigmate du cheveu crépu $^{19}$. Le défrisage travaille ce stigmate. Il I'efface provisoirement, jusqu'aux prochaines repousses. Au-delà de sa fonction de rituel de passage pour les femmes, il devient un rituel de la vie quotidienne par la nécessaire réitération du traitement pour qui veut garder ses cheveux lisses. À travers la pratique du défrisage, il y a une recréation de soi valorisée et valorisante qui permet à certains de sortir du rejet de soi, de s'affirmer de manière positive. Le défrisage nous raconte I'histoire d'une socialisation et d'une tentative d'intégration (économique, politique, sexuelle, etc.) par effacement du stigmate. S'agissant des femmes, prises dans une intersectionnalité (sexe, race et classe), le défrisage met en lumière leur condition qu'elles tentent de dépasser notamment par ce moyen. La pratique du défrisage permet la transcendance de la condition de femme noire en y ajoutant cette touche de beauté et de féminité qui lui manqueraient, selon des critères occidentaux. À l'opposé, le défrisage n'apporte pas particulièrement un surcroît de beauté ou de virilité aux hommes noirs. II n'apporte ni n'enlève rien à leur condition de mâle.

Notre corps est le reflet de notre culture, de notre société et de notre temps. Mais il est aussi le miroir de nos représentations dont celles que nous avons de l'homme et de la femme. La distinction sexuelle participe de la construction de notre identité et de notre altérité. L'une comme l'autre sont mouvantes, fluctuantes et hautement subjectives. De par sa plasticité, la pilosité s'avère propice aux symboles et à la retranscription symbolique de la culture. Cependant, une constante demeure, la distinction sexuelle. Les différences pileuses et capillaires en fonction du sexe sont globalement évidentes. C'est l'une des conséquences et l'un des objectifs du processus de socialisation, faire des hommes et des femmes. Le défrisage est un phénomène intéressant à étudier dans la mesure où il est transversal et touche à divers aspects de la vie humaine. En tant que technique du corps, il est le produit et l'instrument d'une culture donnée, la «culture noire». II s'inscrit dans une histoire raciale, culturelle, mais aussi sexuelle. Le défrisage,

${ }^{19}$ Les nappy font de même mais en refusant le défrisage. 
pratiqué ou rejeté, participe de la création d'une identité noire fictive, mais opérante, d'une identité dépendante de la dualité homme/femme.

L'identité noire, aux cheveux crépus ou défrisés, est une construction mentale mouvante et opérante. Elle demeure tributaire de l'expérience de chaque Noir et du contexte spatial, temporel et social. Elle s'alimente des expériences en lien avec la condition noire. En ce sens, l'identité féminine est aussi fictive, mouvante et opérante, dépendante de chaque femme et du contexte. À mon avis, au-delà de la question raciale, le défrisage questionne d'une part, le genre en tant qu'élément discriminant, d'autre part la pression sociale qui presse les femmes noires à se conformer à un modèle de beauté exotique, enfin la place de référent que les hommes conservent en dépit de tout, car, en définitive, en matière capillaire, les hommes doivent « juste » éviter d'avoir l'air féminin.

Eliane Eock Laïfa est Docteur en Sciences humaines et sociales. Chercheuse associée au Laboratoire DynamE - Dynamiques Européennes, Université de Strasbourg, France.

$\equiv$ andru.ae85@gmail.com.

\section{Bibliographie}

1. BROMBERGER, Christian. Trichologiques : une anthropologie des cheveux et des poils. Montrouge : Bayard, 2010.

2. CHAPMAN, Yolanda M. "I am not my hair! Or am I?": black women's transformative experience in their self perceptions of abroad and at home. Thèse (doctorat en Anthropologie) - Georgia State University, Georgie, 2007.

3. EYENE, Christine. L'afro dans le registre identitaire diasporique. Africultures, v. 1, n. 72, p. 118-125, 2008.

4. HERRON, Carolivia ; CEPEDA, Joe. Nappy hair. Toronto : CNIB, 1998.

5. LE BRETON, David. Anthropologie du corps et de la modernité. Paris : PUF, 2011.

6. LESTER, Neal A. Roots that go behind big hair and bad hair day : nappy hair pieces. Children's Literature in Education, v. 30, n. 3, p. 171-183, 1999.

7. MAUSS, Marcel. Les techniques du corps. Sociologie et anthropologie. Paris : PUF, [1950] 2013. 
8. PATTON, Tracey O. Hey girl, am I more than my hair? African American women and their struggles with beauty, body image, and hair. NWSA Journal, v. 18, n. 2, p. 24-51, 2006.

9. PETONNET, Colette. L'Observation flottante. L'exemple d'un cimetière parisien. L'Homme, v. 22, n. 4, p. 37-47, 1982.

10. ROLLAND-DIAMOND, Caroline. Sociohistoire des Black Studies Departments. IdeAs [En ligne], n, 2, 2012. URL : http://journals.openedition.org/ideas/266.

11. ROSADO, Dione, Sybil. Nappy hair in the diaspora: exploring the cultural politics of hair among women of African descent. Thèse (doctorat en Philosophie). Université de Floride, Floride, 2007.

12. SALIBA, Jacques. Le corps et les constructions symboliques. Socioanthropologie [en ligne], n. 5, 1999. URL : http://socio-anthropologie.revues. org/4.

13. SANT'ANNA, Denise B. de. Être belle au Brésil. Communications, n. 60, p. 95-108, 1995.

14. SARDAN, Jean-Pierre O. de. Émique. L'Homme, v. 38, n. 147, p. 151-166, 1998.

15. SMÉRALDA, Juliette. Peau noire, cheveu crépu : I'histoire d'une aliénation. Pointe-à-Pitre : Éditions Jasor, 2004.

16. SMÉRALDA, Juliette. Du cheveu défrisé au cheveu crépu : de la désidentification à la revendication. Paris : Publibook, 2012.

17. SYNNOTT, Anthony. Shame and glory: A sociology of hair. The British Journal of Sociology, v. 38, n. 3, p. 381-413, 1987.

18. THOMAS, Tiffany. Hair they are: the ideologies of black hair. The York Review, v. 9, n. 1, p. 1-10, 2013.

19. THOMPSON, Cheryl. Black women and identity: What's hair got to do with it? Michigan Feminist Studies, v. 22, n. 1, p. 78-90, 2009.

20. WEITZ, Rose. Women and their hair: seeking power through resistance and accommodation. Gender \& Society, v. 15, n. 5, p. 667-686, 2001.

21. WILKERSON, Kamina. The natural hair movement. Continuum : The Spelman Undergraduate Research Journal, v. 1, n. 1, p. 56-63, 2017. 